\title{
Characterization of chikusaku-eki and mokusaku-eki and its inhibitory effect on sapstaining fungal growth in laboratory scale
}

\author{
${ }^{1}$ N. Velmurugan; ${ }^{1}$ S. S. Chun; ${ }^{1}$ S. S. Han; ${ }^{1,2 *}$ Y. S. Lee \\ ${ }^{1}$ Department of Forest Science and Technology, Institute of Agriculture and Life Sciences, Chonbuk National \\ University, Jeonju, Chonbuk 561-756, Republic of Korea \\ ${ }^{2}$ Institute of Agricultural Science and Technology, Chonbuk National University, Jeonju, Chonbuk 561-756, \\ Republic of Korea
}

Received 21 June 2008; $\quad$ revised 18 August 2008; accepted 25 November 2008; $\quad$ aval(Dble online 10 December 2008

\begin{abstract}
Chikusaku-eki and Mokusaku-eki are natural resources and acidic liquid by-products of bamboo and broad leaved trees charcoal burner. These products contain more than 200 ingredients, including phenols, poly phenols and acetic acids. These by-products were tested for their fungicidal activity against sapstaining fungi. There are no studies about the antifungal activity of Chikusaku-eki and Mokusaku-eki against wood staining fungi in the literature. According to the recent findings, this is the first report about the antifungal activity of Chikusaku-eki and Mokusaku-eki against wood staining fungi. These extracts were more effective against sapstaining fungi at minimum concentrations (0.10-1.0 \%) used in $2 \%$ (malt extract agar) medium. Three Chikusaku-eki (Chikusakueki-I, Chikusaku-eki-II and Chikusaku-eki-III) and 2 Mokusaku-eki (Mokusaku-eki-I, Mokusaku-eki-II) extracts were tested against 4 sapstaining fungal samples to evaluate the inhibition range on sapstaining fungal growth. Ophiostoma flexuosum, Ophiostoma tetropii, Ophiostoma polonicum and Ophiostoma ips were the sapstaining fungi used in this study against bamboo and wood extracts. The initial calibration of extracts was done by calculating the specific gravity, tar calculation and total organic content. The chloroform fractions of these extracts were analyzed by gas chromatographymass spectrometry and both the extracts contained 2,6 dimethoxy phenol, dehydroacetic acid and 2,3,5 trimethoxytoluene. Results revealed that compounds of Chikusaku-eki and Mokusaku-eki markedly inhibited fungal growth at lower concentration. The Chikusaku-eki and Mokusaku-eki have both antifungal, antioxidant properties and a potential to be used as natural preservative in wood industries. Wood slice tests displayed the effective role of both extracts in laboratory level.
\end{abstract}

Key words: Bamboo vinegar, wood vinegar, wood staining fungi, physico-chemical properties, phenolic compounds, antifungal activity

\section{INTRODUCTION}

Sapstaining fungi causes discoloration of wood and creates a significant economic loss to wood industries worldwide. Many control strategies are used to inhibit sapstaining fungi on wood in forest industries, including chemical and biological protection methods. Bamboo charcoal and wood charcoal extracts are favorable natural resource materials to be used as antifungal agents in wood industries. Both the products are acidic and dark brown liquid by-products of the charcoal burner. Bamboo charcoal extract (Chikusakueki) is also called as bamboo vinegar and broad leaved trees charcoal extract (Mokusaku-eki) called as wood vinegar. These extracts are diluted with water and used as disinfection, antibacterial and deodorization

\* *Corresponding Author Email: ysoolee@chonbuk.ac.kr Tel.: +8263 270 2622; Fax: +8263 2702631 materials in the field of agriculture, horticulture, medical and civil engineering (Imamura, 2007). In Japan, Chikusaku-eki is mainly used for folk remedies for the treatment of scabies, eczema, atopic dermatitis and other skin diseases (Kimura et al., 2002).

Lu et al., (2007) studied the inhibition efficiency of a mixed solution of bamboo vinegar and chitosan against soil fungus while the antifungal activity of both Mokusaku-eki and Chikusaku-eki are still not studied well. The microbial growth inhibition by bamboo vinegar and wood vinegar has already been reported (Yatagai and Unrinin, 1987; Sulaiman et al., 2005).

Bamboo extract is mainly prepared from Phyllostachys pubescens, Phyllostachys bambusoides, Phyllostachys heterocycla and Phyllostachys nigra. Broad leaved trees extract is prepared from Quercus serrata, Castanea 
crenata and Prunus jamasakura (Kimura et al., 2002; Imamura, 2007). The main components of both bamboo extract and wood extract are acetic acid and water contributing to 3.5-4 \% and 80-90\%, respectively and more than 200 ingredients are present in these extracts, including phenolic and polyphenolic compounds (Imamura, 2007). Among these ingredients, some have strong antifungal activity such as guaiacol, cresol, 4ethyl-2-methoxyphenol and 2,6-dimethoxyphenol and some have carcinogenic activity such as woodcreosote, benzo[a] pyrene, benz [a] anthracene and 3-methlcholanthrene (Ikegami et al., 1998).

The antifungal activity of both extracts has not yet been studied well. According to the recent knowledge, this is the first approach to evaluate the anti sapstaining activity of both extracts in laboratory level. Five different samples of extracts (including 3 bamboo extracts and 2 broad leaved tree extracts) were obtained from different companies and tested against 4 sapstaining fungal samples. The present work was carried out to investigate the antifungal activity of Chikusaku-eki and Mokusaku-eki against sapstaining fungi in laboratory scale and search for the major compounds present in the extracts with antifungal properties.

\section{MATERIALS AND METHODS}

Fungal samples

The sapstaining fungal samples included: Ophiostoma flexuosum (363175), Ophiostoma tetropii (363182), Ophiostoma polonicum (343181) and Ophiostoma ips (363176). All the fungal samples were obtained from CABI, Bioscience and UK Centre formerly called as International Mycological Institute (IMI). The fungal cultures were grown in $2 \%$ (MEA) malt extract agar medium (Becton, Dickinson and Company, USA) as pre-inoculums at $25^{\circ} \mathrm{C}$ for 4-7 days.

\section{Chikusaku-eki and Mokusaku-eki}

Samples were collected from different parts of South Korea. Table 1 shows detailed information of sources and physiological properties of both Chikusaku-eki and Mokusaku-eki. Chikusaku-eki and Mokusaku-eki were commonly derived from steel batch type kiln. The extracts were generally collected around $200 \sim 600^{\circ} \mathrm{C}$.

The physico-chemical properties of extracts were calculated based on the methods of Mun et al. (2007).

The initial physico-chemical properties of extracts were determined by the following basic properties: $\mathrm{pH}$, the specific gravity, the tar calculation and total organic content percentage. $\mathrm{pH}$ was measured using $\mathrm{pH}$ meter (HI 98160, HANNA instruments). Specific gravity was measured using a glass hydrometer at $15 \pm 0.1^{\circ} \mathrm{C}$.

Appropriate amount $(25 \mathrm{~mL})$ of extracts was taken in a clean crucible and heated in dying oven (FO-600M, JEIO TECH) and then residues obtained after evaporation of volatile compounds were used to calculate the tar content. Total organic content (TOC) was calculated by standard method as described by Mun et al. (2007) and $1 \%$ of (v/v) extracts were titrated using $0.1 \mathrm{~N} \mathrm{NaOH}$ solution. The end point of acetic acid has been determined to be the major organic content in the extracts. TOC was calculated from the volume of the titrated $\mathrm{NaOH}$ at $\mathrm{pH}$ 8.0. The colors of extracts were recorded by naked vision.

\section{Antifungal activity assay}

Inhibition percentage of sapstaining fungi was measured by radial mycelial growth method in presence of various concentrations of Mokusaku-eki and Chikusaku-eki. Five different concentrations (0.001, 0.01, $0.10,0.50$ and $1.00 \%$ ) of both bamboo and broad leaved trees extracts were used in antifungal activity test. The dilution was directly made from the crude extract. Two percentage MEA media was prepared with above mentioned percentages of bamboo and broad leaved trees extracts, separately. Filter sterilization of extracts was performed before adding into media. $6 \mathrm{~mm}$, agar plugs from the pre-inoculum of Ophiostoma flexuosum, $O$. tetropii, O. polonicum and $O$. ips were transferred into $2 \%$ MEA amended with 0.001, 0.01, 0.10, 0.50 and $1.00 \%$ of Mokusaku-eki and Chikusaku-eki. Control plates for all 4 fungal samples were maintained with out extracts. Plates were incubated at $20^{\circ} \mathrm{C}$ for 7 days. The observation of mycelial growth was followed until 7 days and data was registered.

\section{Statistical analysis}

Four replicates were maintained for 4 fungal samples at all concentrations under control. The difference between the mycelial growths of replicates was not significant, thus the average was used for antifungal index analysis. Antifungal index was calculated based on the method of Zhong et al. (2007):

Antifungal index $(\%)=(1-\mathrm{Dt} / \mathrm{Dc}) \times 100$

Where, $\mathrm{Dt}=$ diameter of mycelial growth zone in test 
plate; $\mathrm{Dc}=$ diameter of mycelial growth zone in control plate. Results with significant difference $(P<0.05)$ were considered statistically (Ramos et al., 1997).

\section{GC-MS analysis}

Chikusaku-eki-II (CE-II) sample and Mokusaku-ekiII (ME-II) sample with high fungicidal potential were selected to perform the gas chromatography-mass spectrometry (GC-MS) analysis. Fungicidal potential was determined based on the results of radial growth method. The samples were prepared for GC-MS analysis by following procedure. $500 \mu \mathrm{L}$ of sample was mixed with $1 \mathrm{~mL}$ of chloroform by vortex followed by centrifugation at $13000 \mathrm{rpm}$ for $10 \mathrm{~min}$. The chloroform extract was separated and $1 \mu \mathrm{L}$ of sample was injected into a gas chromatograph (GC HP 6980 Series, Hewlett Packard, USA). Separation of compounds was achieved using a DB-5 Column, J and W Scientific Company (30 $\mathrm{m}$ length, $0.25 \mathrm{~mm}$ ID, $0.25 \mu \mathrm{m}$ film thickness). The oven temperature was elevated from $90{ }^{\circ} \mathrm{C}$ to $300^{\circ} \mathrm{C}$ at the rate of $10^{\circ} \mathrm{C} / 10 \mathrm{~min}$; the final temperature at $300^{\circ} \mathrm{C}$ was maintained for $5 \mathrm{~min}$. The MS was operated in electron ionization (EI) mode at $70 \mathrm{eV}$. Interface temperature was kept at $230^{\circ} \mathrm{C}$. The compound peaks were identified in comparison with available standards.

Evaluation of antifungal activity of Chikusaku-eki and Mokusaku-eki in wood slices in laboratory condition

This experiment was carried out to evaluate the antifungal activity of Chikusaku-eki and Mokusakueki on wood slices under laboratory condition. Pinus radiata was used as wood element. Uncontaminated Pinus ratidata wood block was used to prepare the wood slice in Chonbuk National University Wood Centre. The wood blocks were selected randomly. The slices were placed in sterilized container and then taken to the laboratory. The samples were alienated into small pieces by a sterilized knife (approximately $10 \mathrm{~mm} \times 10$ $\mathrm{mm}$ in size) and then sterilized at $121^{\circ} \mathrm{C}$ for $20 \mathrm{~min}$.

Sterilized wood pieces were soaked with both chikusaku-eki and mokusaku-eki at different concentrations $(0.25,0.5,1.0 \%)$ for $5 \mathrm{~min}$ and then air dried in inside laminar air flow chamber, while control was suspended in sterilized distilled water. Fungal samples as Ophiostoma flexuosum, Ophiostoma tetropii, Ophiostoma polonicum and Ophiostoma ips were inoculated in $2 \%$ MEA plates before placing wood slices into the plates. Control piece and 3 concentrations of the same sample were placed in four sides of each fungus, separately.

\section{RESULTS AND DISCUSSION}

Physicochemical properties of extracts

The physical properties of Chikusaku-eki and Mokusaku-eki were shown in Table 1 . The values of $\mathrm{pH}$ of CE-I, CE-II, CE-III, ME-I and ME-II were 3.0, 3.25, 3.25, 3.5 and 3.5, respectively. The total organic acid contents were 9.87, 11.37, 11.22, 8.90 and $11.07 \%$, respectively. Acidic values and higher specific gravity of the extracts were determined in higher extraction temperature. The specific gravity and tar content percentage were also included in Table 1 . The specific gravity values greatly vary if an iron kiln or combustion furnace is used other than an earthen kiln.

The total organic contents were varied based on wood species and extraction conditions. High organic acid content extract exhibited more antifungal potential than other extracts. It supports that the antifungal activity of extracts was arising from phenolic compounds.

Table 1: Sources and physico-chemical properties of Chikusaku-eki and Mokusaku-eki

\begin{tabular}{|c|c|c|c|c|c|c|c|}
\hline Samples & Wood source & Company & pH & $\begin{array}{l}\text { Specific } \\
\text { gravity }\end{array}$ & Color & $\operatorname{Tar}(\%)$ & $\begin{array}{l}\text { Total organic } \\
\text { content (\%) }\end{array}$ \\
\hline CE-I & $\begin{array}{l}\text { Phyllostachys } \\
\text { bambusoides }\end{array}$ & $\begin{array}{l}\text { Sacheon city wood } \\
\text { industry }\end{array}$ & 3.0 & 1.020 & $\begin{array}{l}\text { Dark } \\
\text { brown }\end{array}$ & 0.59 & 9.87 \\
\hline CE-II & $\begin{array}{l}\text { Phyllostachys } \\
\text { pubescens }\end{array}$ & $\begin{array}{l}\text { Bamboonara wood } \\
\text { company, Damyang }\end{array}$ & 3.25 & 1.029 & $\begin{array}{l}\text { Dark } \\
\text { brown }\end{array}$ & 0.73 & 11.37 \\
\hline CE-III & Phyllostachys nigro & $\begin{array}{l}\text { Bamboonara wood } \\
\text { company, Damyang }\end{array}$ & 3.25 & 1.015 & $\begin{array}{l}\text { Dark } \\
\text { brown }\end{array}$ & 0.54 & 11.22 \\
\hline ME-I & Quercus acutissima & $\begin{array}{l}\text { Sacheon city wood } \\
\text { industry }\end{array}$ & 3.5 & 1.010 & $\begin{array}{l}\text { Light } \\
\text { brown }\end{array}$ & 0.27 & 8.90 \\
\hline ME-II & Quercus serrata & $\begin{array}{l}\text { Jin-Jeop town wood } \\
\text { industry }\end{array}$ & 3.5 & 1.005 & $\begin{array}{l}\text { Dark } \\
\text { brown }\end{array}$ & 0.30 & 11.07 \\
\hline
\end{tabular}


Inhibitory effect on sapstaining fungal growth

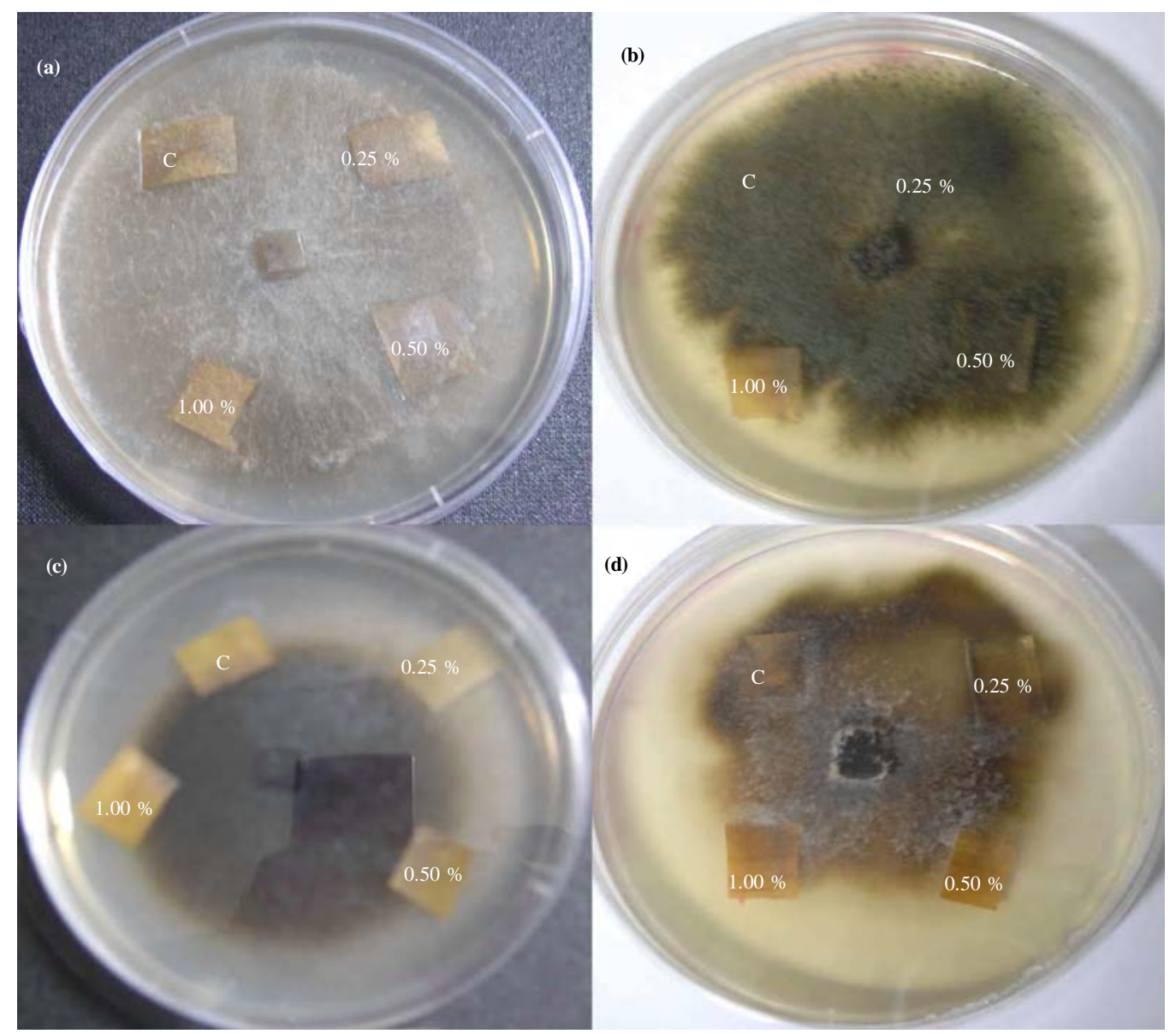

Fig. 1: Inhibition of (a) Ophiostoma tetropii, (b) Ophiostoma polonicum, (c) Ophiostoma ips and (d) Ophiostoma flexuosum on wood slices treated by different concentrations of Chikusaku-eki (CE-II)

Thus, those extracts (CE-II and ME-II) were selected for further studies.

\section{Antifungal activity of Chikusaku-eki}

The antifungal activities of CE-I, CE-II and CE-III were assessed against sapstaining fungi. Table 2 shows the antifungal activity of extracts in $2 \%$ MEA medium.

Among 3 samples of Chikusaku-eki, CE-II and CE-III were very active since all four tested sapstaining fungi were completely inhibited when $1.0 \%$ used.

Same time minimum growth of all tested fungi was observed in $1.0 \%$ of CE-I sample containing plates.

0.5 of CE-II and CE-III was completely inhibited the growth of Ophiostoma flexuosum (Table 2) and $0.5 \%$ of CE-II completely inhibited the growth of Ophiostoma tetropii and Ophiostoma ips (Table 2). CE-I was not so active against sapstaining since mycelial growth of all tested sapstaining fungi was observed in all concentrations of extract. Ophiostoma polonicum (Table 2) shows high tolerant activity against all 3 extracts with indicates equal growth to control in presence of 0.001, 0.01 and $0.10 \%$ extract. Growth of Ophiostoma tetropii and Ophiostoma ips were slightly inhibited even minimum concentration of $0.001 \%$ used (Table 2). Thus, Ophiostoma tetropii and Ophiostoma ips are highly sensitive towards Chikusaku-eki.

\section{Antifungal activity of Mokusaku-eki}

Compare to Chikusaku-eki, Mokusaku-eki was not highly active extract against sapstaining fungi. All samples of Mokusaku-eki have not completely inhibited the mycelial growth of tested sapstaining fungi even high concentration of $1.0 \%$ used. Ophiostoma flexuosum shows (Table 2) high resistance 
Int. J. Environ. Sci. Tech., 6 (1), 13-22, Winter 2009

Table 2: Antifungal activity of Chikusaku-eki and Mokusaku-eki (\% inhibition)

\begin{tabular}{|c|c|c|c|c|c|c|c|c|c|c|c|c|c|c|c|}
\hline \multirow{3}{*}{ Fungal samples } & \multicolumn{15}{|c|}{ Antifungal index (\%) } \\
\hline & \multicolumn{5}{|c|}{0.001} & \multicolumn{5}{|c|}{0.01} & \multicolumn{5}{|c|}{0.1} \\
\hline & CE-I & CE-II & CE-III & ME-I & ME-II & CE-I & CE-II & CE-III & ME-I & ME-II & CE-I & CE-II & CE-III & ME-I & ME-II \\
\hline O. flexuosum & 0 & 0 & 0 & 0 & 0 & 5 & 5 & 5 & 0 & 0 & 20 & 30 & 5 & 0 & 0 \\
\hline O. tetropii & 5 & 5 & 3 & 0 & 5 & 5 & 10 & 7 & 5 & 5 & 17 & 46 & 34 & 7 & 10 \\
\hline O.polonicu & 0 & 0 & 0 & 0 & 0 & 0 & 0 & 0 & 0 & 0 & 0 & 11 & 11 & 0 & 11 \\
\hline O. ips & 12 & 17 & 19 & 12 & 12 & 12 & 17 & 19 & 12 & 12 & 19 & 44 & 36 & 14 & 17 \\
\hline \multirow[t]{2}{*}{ Fungal samples } & & & & & 0.5 & & & & & 1.0 & & & & & \\
\hline & CE-I & CE-II & CE-III & ME-I & ME-II & CE-I & CE-II & CE-III & ME-I & ME-III & & & & & \\
\hline O. flexuosum & 10 & 100 & 100 & 10 & 15 & 20 & 100 & 100 & 30 & 50 & & & & & \\
\hline O. tetropii & 30 & 100 & 76 & 24 & 27 & 41 & 100 & 100 & 49 & 39 & & & & & \\
\hline O. polonicu & 11 & 56 & 45 & 11 & 33 & 27 & 100 & 100 & 33 & 39 & & & & & \\
\hline O. ips & 25 & 100 & 78 & 28 & 31 & 47 & 100 & 100 & 39 & 53 & & & & & \\
\hline
\end{tabular}

activity towards Mokusaku-eki. Complete growth of Ophiostoma flexuosum was observed in 0.001, 0.01 and $0.10 \%$ of Mokusaku-eki used media (Table 2). An overall result of Mokusaku-eki shows that effects of Mokusaku-eki were not significant (Table 2).

Thus, Mokusaku-eki is not suitable product for inhibition of sapstaining fungi in wood industries.

\section{Chemical composition analysis of CE-II and ME-II by $G C-M S$}

The main compounds of extracts were identified by GC-MS. A number of 6 spectral peaks were detected in GC-MS analysis of CE-II. These peaks were detected at retention time (RT) 6.64, 8.36, 9.68, 10.69, 11.97 and 12.97 min (Table 3). Among these 6 peaks, 5 peaks were identified by mass spectroscopy (MS); these compounds may responsible for antifungal activity of CE-II. The compounds are 2methoxy phenol (6.64), 2-methoxy-4-methyl phenol (8.36), 2,6-dimethoxy phenol (10.69), dehydroacetic acid (11.97) and 2,3,5-trimethoxytoluene (12.97). GCMS analysis of chloroform fraction of ME-II revealed four well defined spectral peaks (Table 3); compounds 1, 2, 3 and 4 were detected at RT 10.70, 11.97, 12.98 and $13.98 \mathrm{~min}$. The major peak was obtained in RT of $10.70 \mathrm{~min}$. The compounds of these four peaks may contain high antifungal activity among other ingredients of ME-II. Thecompounds were identified as 2,6-dimethoxy phenol, dehydroacetic acid, 2,3,5-trimethoxy toluene and 5hydroxy-3-oxo-2-propionyl-1gamma-1-acetone-4heptnoic acid by MS analysis.
Antifungal activity of Chikusaku-eki (CE-II) and Mokusaku-eki (ME-II) in wood slices

Antifungal activity of Chikusaku-eki and Mokusaku-eki were determined on wood slices under laboratory condition (Fig. 1). Control wood slices were completely occupied by all the tested fungal samples after 7 days.

Growth of Ophiostoma polonicum and Ophiostoma flexuosum hypha was inhibited on wood slices treated with $1.0 \%$ CE-II (Fig. 1 a and d). While Ophiostoma tetropii hypha growth was observed in wood slice treated with $1.0 \%$ CE-II (Fig. 1 a). Thus, the experiment supports the results of radial mycelial growth inhibition test. As mentioned previously, the antifungal activity of Mokusaku-eki is lesser than Chikusaku-eki. All the tested fungi (except Ophiostoma flexuosum) were grown in wood slices treated with $1.0 \%$ of Mokusakueki. Developing an eco friendly product is essential for the inhibition of fungal growth on wood and wood protection. Both Mokusaku-eki and Chikusaku-eki have been recognized as highly active extracts with antimicrobial activity (Rouha et al., 2000; Kimura et al., 2002) and both are natural by-products. In this study, the antifungal activity of Chikusaku-eki and Mokusakueki were studied from minimum possible concentration to high concentration. To determine the chemical composition of Chikusaku-eki and Mokusaku-eki, the chemical analysis was conducted by GC-MS spectrum (Figs. 2 and 3). The results showed the content of both acetic acid and phenolic compounds in Chikusaku-eki and Mokusaku-eki. GC-MS results showed a higher content of 2,6-dimethoxy phenol in both Chikusakueki (34.72 \%) and Mokusaku-eki (58.06 \%). 
$N$. Velmurugan et al.

Table 3: Major compounds in Chikusaku-eki (CE-II) and Mokusaku-eki (ME-II) identified by GC-MS spectrum

\begin{tabular}{|c|c|c|c|}
\hline & & \multicolumn{2}{|c|}{ Area (\%) } \\
\hline $\mathrm{RT}(\min )$ & Compounds & Mokusaku-eki -2 (ME-II) & Chikusaku-eki-2 (CE-II) \\
\hline 6.63 & 2-methoxy phenol & - & 16.55 \\
\hline 8.36 & 2-methoxy-4-methyl phenol & - & 11.37 \\
\hline 10.69 & 2,6-dimethoxy phenol & 58.06 & 34.72 \\
\hline 11.97 & dehydroacetic acid & 18.60 & 21.89 \\
\hline 12.97 & 2,3,5-Trimethoxy toluene & 18.19 & 15.47 \\
\hline 13.98 & $\begin{array}{l}\text { 5-hydroxy-3-oxo-2-propionyl- } \\
\text { 1gamma-1-acetone-4-heptnoic acid }\end{array}$ & 5.15 & - \\
\hline
\end{tabular}

Dehydroacetic acid comprises $21.89 \%$ of Chikusakueki and $18.60 \%$ of Mokusaku-eki. As the results of GC analysis, the total phenolic content was $58.06 \%$ in Mokusaku-eki and $62.64 \%$ in Chikusaku-eki.

According to previous research works, both the extracts were mostly composed of 80 to $90 \%$ water and 10 to $20 \%$ of more than 200 ingredients. Major compounds of these ingredients are organic acids such as acetic acid and propionic acid. The total organic content of extracts varies and depends on the wood species, mostly around 3-4 \% in bamboo and 2-3 \% in broad leaved trees (Mun et al., 2007). Among these organic compounds acetic acid was recognized as the major component, approximately $59 \%$ in bamboo extract and 45-69 \% in broad leaved trees (Mun et al., 2007).

Mokusaku-eki and Chikusaku-eki also contain phenolic compounds as major constitutes. The concentration of phenolic compounds also vary depending on wood species about $0.53 \%$ in bamboo extract and 0.16 to $0.15 \%$ in broad leaved trees extract.

Panasenko (1967) and Land et al. (1987) reported that $\mathrm{pH}$ tolerant of sapstaining fungi are more and range between $\mathrm{pH} 2$ to $\mathrm{pH} 10$. The phenolic compounds were identified and indicated as central efficacy tested against sapstaining fungal growth from 0.25 to $1.00 \%$ where the sapstaining fungal growth was completely inhibited in all concentrations (data not shown).

Presence of phenolic compounds in both extracts was proved by GC-MS analysis in the present study. Based on the antifungal activity results and GC analysis results, complex mixture of phenolic compounds was responsible for antifungal activity of Mokusaku-eki and Chikusaku-eki. Since the concentration of organic acids are almost similar in both extracts, in reference to Mun et al. (2007) the concentration of phenolic compounds in Chikusaku- eki was 3-4 folds higher than Mokusaku-eki. The antifungal activity tests also had shown the fewer antifungal activity of Mokusaku-eki in all concentrations. Antifungal and antimicrobial activities of plant phenols were studied well (Pereira et al., 2007; Prats et al., 2003; Markin et al., 2002). The exact mode of action of antifungal and antimicrobial activities of wood phenols yet not studied well.

The peaks detected in RT at 10.70, 11.97, 12.98 and $13.98 \mathrm{~min}$ for Mokusaku-eki and at 6.64, 8.36, 9.68, 10.69, 11.97 and 12.97 min for Chikusaku-eki were considered to be compounds with antifungal activity. During the preparation of wood extracts, the phenols, guaiacol and their derivatives were arising from thermal degradation of wood lignin (Maria et al., 2002). These compounds have been considered as responsible for antioxidant and antimicrobial activity (Maria et al., 2002). Some authors reported the antioxidant activities of 2-methoxyphenol (guaiacol), 2,6-dimethoxyphenol (syringol) present in wood extract (Ogata et al., 1997; Kajiyama and Ohkatsu, 2001; Bortolomeazzi et al., 2007). Since cell membrane acts as selective barrier for the passage of solution between cytoplasm and cell surface area, phenols can easily interact with cell membrane and damage it. This causes the cell burst and release of cell constituents from cytoplasm which leads the cell death (Park et al., 2001).

The antifungal ability of phenolic compounds are depends on the fungal enzyme inhibition which contains SH groups in their active sites and the antifungal activity of phenolic compounds might be influenced by the water soluble properties of phenolic compounds (Cowan, 1999). Akgul and Kivanc, (1988) studied the antifungal activity of thymol and carvacrol against Aspergillus flavus, Geotricum candidum, Mucor spp. and Penicillium spp. in PDA agar at 0.0025 


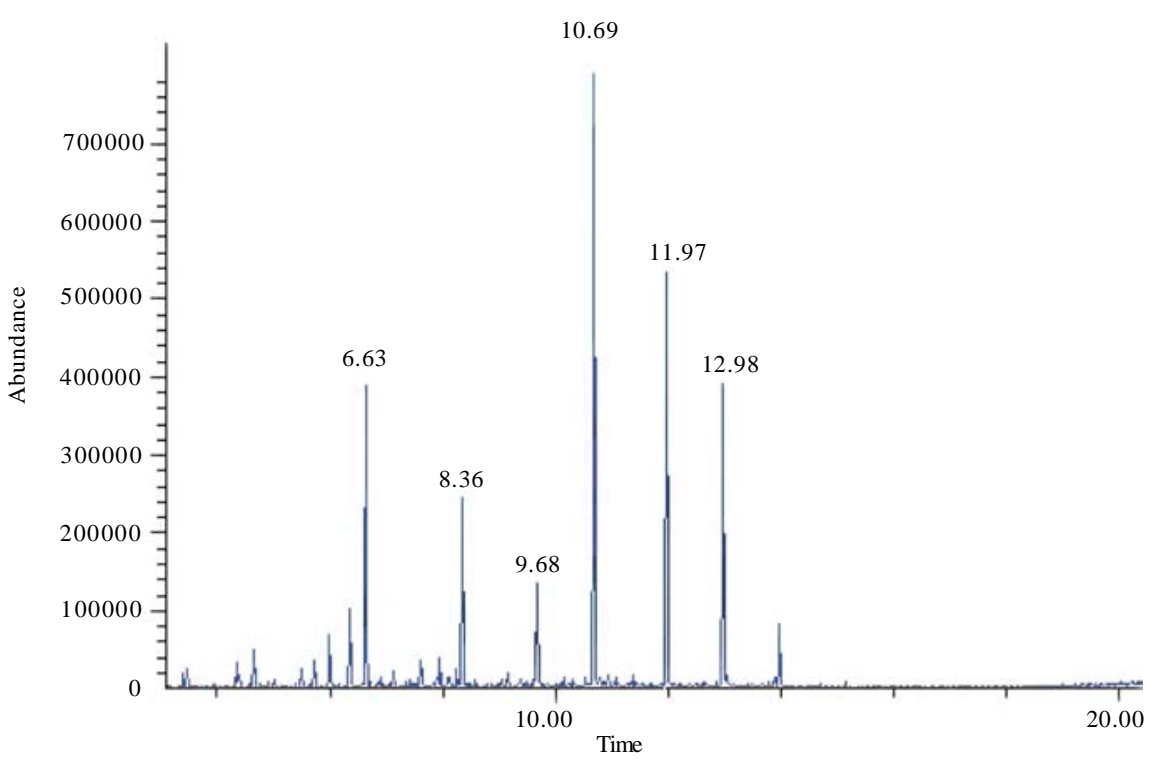

Fig. 2: GC chromatogram of Chikusaku-eki 2 (CE-II) sample; Column DB-5 with 0.25 $\mathrm{mm}$ ID, $0.25 \mu \mathrm{m}$ film thickness used for analysis

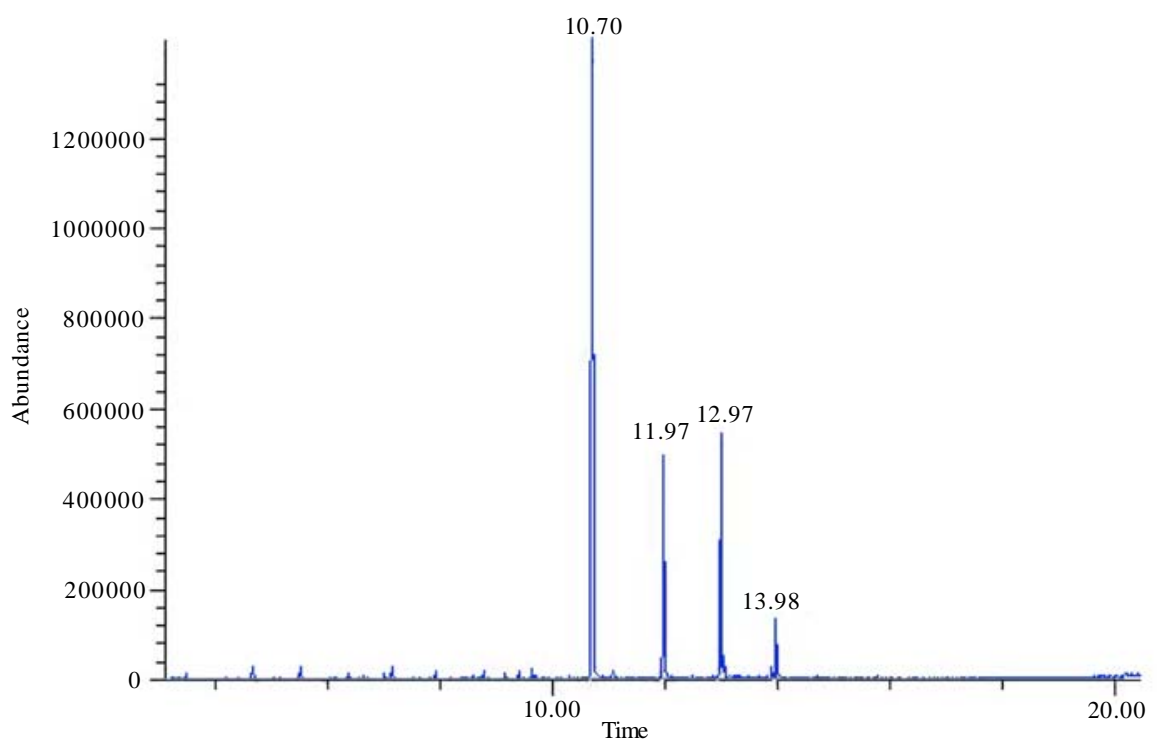

Fig. 3: GC chromatogram of Mokusaku-eki 2 (ME-II) sample; Column DB-5 with $0.25 \mathrm{~mm}$ ID, $0.25 \mu \mathrm{m}$ film thickness used for analysis

and $0.05 \%(\mathrm{~W} / \mathrm{V})$. The complete inhibition was observed by thymol and carvacrol. The small amount of minor compounds in Chikusaku-eki and Mokusakueki might also contribute the antifungal activity.

Phenolic compounds are exhibited higher antimicrobial property than alcohols, aldehydes, ketones, ethers and hydro carbons (Kurita and Koike, 1983). Essential oils present in phenols possessed a significant antifungal activity than essential oils in ketone. The antifungal activities of compounds are mainly based on fungal group and fungal strains (Benjilali et al., 1984). However, multiple mode of action was observed in antifungal mechanism of phenolic compounds. The interaction of phenolic compounds with cell membrane caused the release of intracellular compounds, fatty acid change, 
phospholipid constituent, impair the energy metabolism, alter in nutrient uptake, electron transport and influence in genetic material synthesis (Nychas, 1995). The antioxidant capacity of phenolic compounds depends on the chemical structure of the molecule and the ability of a compound to act as a reducing agent and thus to function as an antioxidant (Bortolomeazzi et al., 2007) and antifungal activity mainly based on the fungal enzyme inhibition by the oxidized compounds through protein nonspecific interaction or reaction with sulfhydryl groups (Voda et al., 2004). The hydroxyl group of phenolic compounds can easily react with enzymes and form hydrogen bonds. These bonding formations can extent the inhibition of phenolic compounds (Farag et al., 1989). The antifungal activity of phenolic antioxidants also depends on the degree of steric hindrance, the lipid solubility and the location of hydroxyl group on the molecule (Raccach, 1984). In addition, the antioxidant performance could be due to the effect of the $\mathrm{pH}$ on the ionization of the phenolic compounds (Borotolomeazzi et al., 2007). At the $\mathrm{pH}$ of 7.01, the hydroxyl group of phenolic compound is not ionized. However, at pH 3.0, the phenolic hydroxyl group are ionized and antioxidant activity also are increased (Borotolomeazzi et al., 2007).

This hypothesises was observed for guaiacol (Borotolomeazzi et al., 2007). The electron-attractive inductive effect of the alpha carbonyl group in the para position should in fact destabilize the phenoxyl radical and reduce the radical scavenging ability of these compounds (Kajiyama and Ohakatsu, 2007). The previous reports showed that electron donor or bulky groups at the ortho or para group of the phenol compounds were required for the inhibition of lipid oxidation. The lipid oxidation was found to inhibit the fungal growth (Vaughn and Gardner, 2003). The allyl groups, which contain phenolic compounds, are effective antioxidants because of the $\mathrm{O}_{2}$ and hydroxyl radical scavenging ability. However, the phenolic compounds without allyl groups also play a role in the termination of free radical chain reactions. The antifungal activity of guaiacol is enhanced by the presence of alkyl group(s) on its benzene ring and ether compounds enhanced with methyl group (Kurita et al., 1981). Voda et al., (2004) reported that the most hydrophobic compounds were more active and the least hydrophobic compounds had least activity against fungal growth . Voda et al., (2004) also suggested that compounds, which contained only one oxygen ring in the aromatic ring, had more antifungal activity compare to compounds with two to three oxygen groups. Cresol, eugenol and isoeugenol were more active antifungal compounds since aromatic ring of these compounds had oxygen group in second position and hydrocarbon groups in the fourth position (Voda et al., 2004). The antifungal activity also influenced by size of hydrocarbon group; large hydrocarbon groups are more active than compounds which contain smaller hydrocarbon groups (Voda et al., 2004). Thus, it can be suggested that fungicidal responsibility of Chikusaku-eki and Mokusaku-eki is rising from its ingredients such as phenols, polyphenols and acetic acid with influence of $\mathrm{pH}$ of extracts and the fungicidal responsibility depends on the following reasons:

Contact between cell surface and phenolic compounds; Intake of phenolic compounds into inside of fungal cell and reaction between the phenolic compounds and target molecule. The uptake of phenolic compounds may be influenced by some molecules like proteins.

Creosote, creosote solutions and oil borne preservatives are universally used as chemical preservatives to protect wood from staining fungi and other decay fungi. These chemicals preservatives should not be used at homes or other living areas because of toxic fumes and carcinogenic effects on plants, animals and humans (Chirra, 1995). Kimura et al. (2002) found that Chikusaku-eki do not have carcinogenic effects in cell systems. Chikusaku-eki and Mokusaku-eki are diluted with water and used as a folk medicine for skin disease in Japan. Consequently, it can be used in wood industries without carcinogenic effects. Since it is a natural resource, compare to the wood preservatives synthesized chemically, the material used in the present study is much safe as it is a consortium of many materials and it's concentration is much lower. On the other hand, application of Chikusaku-eki and Mokusaku-eki as wood preservatives can be viewed as an friendly environment waste management strategy because it is available as waste in large quantities from charcoal producing industries. These extracts could be used at homes or other living areas since diluted extracts are directly consumed as folk medicine for skin disease. Chikusaku-eki and Mokusaku-eki are natural resource materials, easily 
available and inexpensive. Therefore, it is favorable fungicide for wood industries.

\section{CONCLUSION}

This investigation has given preliminary information to determine the antifungal activity of Mokusaku-eki and Chikusaku-eki against sapstaining fungi and influence of $\mathrm{pH}$, phenolic compounds and other ingredients in fungicidal activity in laboratory level.

Further investigations are currently conducted to find out the efficacy of Mokusaku-eki and Chikusakueki as an antifungal agent on wood in the field. Also, further studies are required to find out the exact mode of phenolic compounds action against fungal growth.

These extracts are easily available, inexpensive and non-toxic to environment which validate their applications in wood industries.

\section{ACKNOWLEDGEMENTS}

This study was supported by Technology Development Program for Agriculture and Forestry, Ministry of Agriculture and Forestry, Republic of Korea (No. 105070-03-3-SB010).

\section{REFERENCES}

Akgul, A.; Kivanc, M., (1988). Inhibitory effects of selected Turkish spices and oregano compounds on some food borne fungi., Int. J. Food Microbiol., 6 (3), 263-268.

Benjilali, B.; Tan Taoui Elaraki, A.; Ayadi, A.; Ihlal, M., (1984). Methods to study antimicrobial effects of essential oils: Application to the antifungal activity of six Moroccan essences., J. Food Protect., 47 (10), 748-752.

Bortolomeazzi, R.; Sebastianutto, N.; Toniolo, R.; Pizzariello, A., (2007). Comparative evaluation of the antioxidant capacity of smoke flavouring phenols by crocin bleaching inhibition, DPPH radical scavenging and oxidation potential., Food Chem., 100 (4), 1481-1489.

Chirra, F., (1995). Ohio pesticide application training,. in: Wood preservation student workbook. The Ohio State University Extention. Product Code 821-13.

Cowan, M. M., (1999). Plant products as antimicrobial agents., Clini. Micro. Rev., 12 (4), 564-582.

Farag, R. S.; Daw, Z. Y.; Hewedi, F. M.; El baroty, G. S. A., (1989). Antimicrobial activity of some Egyptian spice essential oils., J. Food Protect., 52 (9), 665-667.

Ikegami, F.; Sekine, T.; Fujii, Y., (1998). Antidermaptophyte acitivity of phenolic compounds in “Mokusaku-eki”., Yakugaku Zasshi, 118 (1), 27-30.

Imamura, E., (2007). Anti-allergy composition comprising wood vinegar- or bamboo vinegar distilled solution. United States Patent 7214393.

Kajiyama, T.; Ohkatsu, Y., (2001). Effect of parasubstituents of phenolic antioxidants., Polym. Degrad. Stability, 71 (3), 445-452.
Kimura, Y.; Suto, S.; Tatsuka, M., (2002). Evaluation of Carcinogenic/Co-carcinogenic activity of chikusaku-eki, a bamboo charcoal by-product used as folk remedy, in BALAB/c 3T3 cells., Biol. Pharm. Bull., 25 (8),10261029 .

Kurita, N.; Koike, S., (1983). Synergistic antimicrobial effects of ethanol, sodium chloride, acetic acid and essential oils., Agr. Biol. Chem., 47 (1), 67-75.

Kurita, N.; Miyaji, M.; Kurane, R.; Takahara, Y., (1981). Antifungal activity of components of essential oils. Agric. Biol. Chem., 45 (4), 945-952.

Land, C. J.; Banhidi, Z. G.; Albertsson, A., (1987). Coldtolerant (psychrotophic) moulds and blue stain fungi from softwood in Sweden, growth rates in relation to $\mathrm{pH}$ and temperature., Nordic J. Botany, 207 (1), 97-106.

Lu, K. C.; Kuo, C. V.; Liu, C. T., (2007). Inhibition efficiency of a mixed solution of bamboo vinegar and chitosan against Ralstonia solanacearum., Taiwan J. Forest Sci., 22 (3), 329-338.

Markin, D.; Duek, L.; Berdicevsky, I., (2003). In vitro antimicrobial activity of Olive leaves. Mycoses, 46 (34), 132-136.

Mun, S. P.; Ku, C. S.; Park, S. B., (2007). Physiochemical characterization of pyrolyzates produced from carbonization of lignocellulosic biomass in a batch-type mechanical kiln., J. Ind. Eng. Chem., 13 (1), 127-132.

Nychas, G. J. E., (1995). Natural antimicrobials from plants, in: Gould, G. W., (Ed.), New Methods of Food Preservation, Blackie Academic Professional, London. 58-89.

Ogata, M.; Hoshi, M.; Shimotohno, K.; Urano, S.; Endo, T., (1997). Antioxidant activity of magnolol, honokiol and related phenolic compounds., J. Am. Oil Chem. Soc., 74 (5), 557-562.

Panasenko, V. T., (1967). Ecology of microfungi., Bot. Rev., 33 (3), 189-215.

Park, E. S.; Moon, W. S.; Song, M. J.; Kim, M, N.; Chung, K. H.; Yoon, J. S., (2001). Antimicrobial activity of phenol and benzoic acid derivatives. Int. Biodeter. Biodegr., 47 (4), 209-214.

Pereira, A. P.; Ferreria, I. C. F. R.; Marcelino, F.; Valentao, P.; Andrade, P. B.; Seabra, R.; Estevinho, L.; Bento, A.; Pereira, J. A., (2007). Phenolic compounds and antimicrobial activity of Olive (Olea europaea L. Cv. Cobrancosa) Leaves., Molecules, 12 (5), 1153-1162.

Prats, E.; Bazzalo, M. E.; Leon, A.; Jorrin, J. V., (2003). Accumulation of soluble phenolic compounds in sunflower capitula correlates with resistance to Sclerotinia sclerotiorum., Euphytica, 132 (3), 321-329.

Raccach, M., (1984). The antimicrobial activity of phenolic antioxidants in foods: A review., J. Food Safety, 6 (3), 141-170.

Ramos, A. C. S.; Dantas Neto, A. A.; Castro Dantas, T. N. C., (1997). Application of an experimental methodology in the optimization of a tungsten concentration process by microemulsions., Braz. J. Chem. Eng., 14 (2), 159165.

Rauha, J. P.; Remes, S.; Heinonen, M.; Hopia, A.; Kahkonen, M.; Kujala, T.; Pihlaja, K.; Vuorela, H.; Vuorela, P., (2000). Antimicrobial effects of finnish plant extracts containing flavonoids and other phenolic compounds. Int. J. Food Microbiol., 56 (1), 3-12. 
Sulaiman, O.; Murphy, R. J.; Hashim, R.; Gritsch C. S., (2005). The inhibition of microbial growth by bamboo vinegar., J. Bamboo Rattan, 4 (1), 71-80.

Vaughn, S. F.; Gardner, H. W., (1993). Lipoxygenase-derived aldehydes inhibit fungi pathogenic on soybean., J. Chem. Ecol., 19 (10), 2337-2345.

Voda, K.; Boh, B.; Vrtacnik, M., (2004). A quantitative structure-antifungal activity relationship study of oxygenated aromatic essential oil compounds using data structuring and PLS regression analysis., J. Mol. Model, 10 (1), 76-84.
Yatagai, M.; Unrinin, G., (1987). By-products of wood carbonization III. Germination and growth acceleration effects of wood vinegars on plant seeds. Mokuzai Gakkaishi, 35 (6), 564-571.

Zhong, Z.; Chen, R.; Xing, R.; Chen, X.; Liu, S.; Guo, Z.; Ji, X.; Wang, L.; Li, P., (2007). Synthesis and antifungal properties of sulfanilamide derivatives of chitosan., Carbohyd. Res., 342 (16), 2390-2395.

\section{AUTHOR (S) BIOSKETCHES}

Velmurugan, N., Ph.D. student, Department of Forest Science and Technology, Institute of Agriculture and

Life Sciences, Chonbuk National University, Jeonju, South Korea. Email: velmmk@gmail.com

Chun, S. S., Ph.D. student, Department of Forest Science and Technology, Institute of Agriculture and Life Sciences, Chonbuk National University, Jeonju, South Korea. Email: ewood@kornet.net

Han, S. S., Ph.D., Associate professor, Institute of Agricultural Science and Technology, Chonbuk National University, Jeonju, South Korea. Email: sshan@chonbuk.ac.kr

Lee, Y. S., Ph.D., Associate professor, Institute of Agricultural Science and Technology, Chonbuk National University, Jeonju, South Korea. Email: ysoolee@chonbuk.ac.kr

This article should be referenced as follows:

Velmurugan, N.; Chun, S. S.; Han, S. S.; Lee, Y. S., (2008). Characterization of chikusaku-eki and mokusakueki and its inhibitory effect on sapstaining fungal growth in laboratory scale. Int. J. Environ. Sci. Tech., 6 (1), 1322. 